\section{International Scientific Journal Theoretical \& Applied Science}

\author{
Vladimir Igorevich Esaulov \\ Institute of psychotherapy and clinical psychology \\ Psychotherapy Department, Lecturer, \\ Assistant, Department of Psychotherapy, \\ Pirogov Russian National Research Medical \\ University, Moscow, Russia \\ v-esaulov@yandex.ru
}

Year: $2016 \quad$ Issue: 12 Volume: 44

Published: $30.12 .2016 \quad$ http://T-Science.org

SECTION 20. Medicine.

\title{
PRACTICE OF INTEGRATIVE PSYCHOTHERAPY OF ANXIETY- PHOBIC DISORDERS WITH IRRITABLE BOWEL SYNDROME PATIENTS
}

\begin{abstract}
IBS) were selected, they have all shown typical for this condition abdominal disorders and anxiety-phobic symptoms. All patients went through psychotherapy sessions along with standard gastroenterological methods of therapy. The treatment course took 3 month and included 6 weeks of base therapy and 6 more weeks of supporting therapy. The techniques that were used included integrative psychotherapy with hypnosuggestive techniques, elements of autotraining and relaxation techniques, neurolinguistic programming and a number of other psychotherapeutic methods. As a result of this combination of therapy methods patients showed lower anxiety level, better state of health and lower abdominal pains at the first month. As a second month of supportive therapy went by the IBS patients condition both psychoemotional and somatic became stable and well.

Key words: irritable bowel syndrome, anxiety-phobic disorders, integrative psychotherapy.

Language: Russian

Citation: Esaulov VI (2016) PRACTICE OF INTEGRATIVE PSYCHOTHERAPY OF ANXIETY-PHOBIC DISORDERS WITH IRRITABLE BOWEL SYNDROME PATIENTS. ISJ Theoretical \& Applied Science, 12 (44): $135-138$.

Soi: http://s-o-i.org/1.1/TAS-12-44-25 Doi: crossef http://dx.doi.org/10.15863/TAS.2016.12.44.25

\section{ПРИМЕНЕНИЕ ИНТЕГРАТИВНОЙ ПСИХОТЕРАПИИ В ЛЕЧЕНИИ ТРЕВОЖНО-ФОБИЧЕСКИХ РАССТРОЙСТВ У ПАЦИЕНТОВ С СИНДРОМОМ РАЗДРАЖЕННОГО КИШЕЧНИКА}

Аннотация: Отобраны 58 пациентов с синдромом раздраженного кишечника (СРК), имеющие типичные для этого расстройства абдоминальные расстройства и тревожно-фобическую симптоматику. Со всеми пациентами проводились психотерапевтические занятия в сочетании со стандартными гастроэнтерологическими методами терапии. Курс лечения продолжался 3 месяца, и включал 6 недель основной терапии, и 6 недель - поддерживающей терапии. Был использован интегративный психотерапевтический подход, включающий гипносуггестивные техники, элементы аутотренинга и приемов релаксачии, нейро-линвистическое программирование, $u$ ряд других психотерапевтических техник. В результате применения такой комбинированной терапии снизилась тревожность, отмечено существенное улучшение самочувствия, уменьшение абдоминальных жалоб уже в течение первого месяца. Начиная со второго месяйа на поддерживающей терапии стало стабильным и благополучным как психоэмоциональное, так и соматическое состояние пациентов с СРК.
\end{abstract}

Ключевые слова: синдром раздраженного кишечника, тревожно-фобические расстройства, интегративная психотерапия.

\section{Введение}

Синдром раздраженного кишечника (СРК) является одним из самых распространенных психосоматических заболеваний желудочнокишечного тракта [1-3]. Большинством современных исследователей признан биопсихосоциальный характер этого расстройства [1,4-7]. В исследованиях возможностей терапевтического воздействия на разные звенья болезни уже много лет принимают участие как врачи-гастроэнтерологи, так и врачипсихиатры и психотерапевты [6-8] .

Вследствие того, что при СРК имеется теснейшее взаимовлияние психической и гастроэнтерологической составляющих болезни, поиски новых эффективных методов лечения 
больных c этой патологией по-прежнему остаются одной из актуальных проблем и современной гастроэнтерологии и пограничной психиатрии [4-8].

У пациентов с СРК можно наблюдать самые различные пограничные психические расстройства, состоящие из разнообразных комбинаций тревожных, депрессивных, астенических, истерических и иных симптомов, делающих картину психоэмонального состояния больных весьма пестрой, изменчивой и полиморфной [1,6-7,9-12].

Среди преобладающих психических расстройств основными являются расстройства тревожно-фобического и тревожнодепрессивного спектра различной интенсивности $[2,7,9,10,12,14]$. При этом часто наблюдается пессимистическая оценка перспектив лечения, неверие в его результативность, снижение веры в себя, часто возникающие тревожные опасения и страхи за свое здоровье, сопровождающиеся различными астено-вегетативными расстройствами [10,12-15].

Лекарственная терапия этих проявлений заболевания антидепрессантами и другими психофармакотерапевтическими препаратами дает хотя и неплохой, но временный эффект, и вызывает необходимость возобновления приема лекарств во время ухудшений, часто приходящихся на периоды сезонных изменений $[3,7,8]$.

К сожалению, прием только лекарственных препаратов не может в полной мере разрешить многие внутренние противоречия, укрепить проблемную самооценку этих пациентов, снять пессимистическую оценку перспектив, убрать многочисленные страхи и ипохондрические переживания у больных без активной психотерапевтической коррекции.

Естественно, при этом требуется подбор персонального подхода к каждому пациенту. Важно создание эффективного альянса врача и пациента, понимание и терпеливое отношение к больному, внимательность и доброжелательность от оказывающих помощь врачей и медперсонала, их умение ободрить и настроить пациента на позитивный лад, укрепить веру в себя, в лечение, в возможность выздоровления. Вследствие этого довольно трудно представить современную терапию пациентов с СРК без включения в лечебный процесс врачей-психотерапевтов или имеющих соответствующую подготовку клинических психологов [15-17].

Целью настоящей работы являлся подбор краткосрочных техник психотерапии, помогающих за относительно непродолжительное время наряду со стандартным лечением СРК стабилизировать как психоэмоциональное, так и соматическое состояние.

Материалы и методы: Для проведения исследования были отобраны 58 пациентов (14 мужчин и 44 женщины, в возрасте 17-55 лет), с синдромом раздраженного кишечника, имеющие типичные для этого расстройства абдоминальные расстройства и тревожно-фобическую симптоматику.

Тревожно-фобическая симптоматика верифицировалась осмотром психиатра. На протяжении всего курса лечения велось динамическое наблюдение, анкетирование, и заполнение пациентами дневников самочувствия.

Со всеми 58 пациентами проводились психотерапевтические занятия в сочетании со стандартными гастроэнтерологическими методами терапии.

Курс лечения продолжался 3 месяца, и включал 6 недель основной терапии, и 6 недель поддерживающей терапии.

Был использован интегративный психотерапевтический подход, включающий гипносуггестивные техники, элементы аутотренинга, релаксационные упражнения, нейро-линвистическое программирование, техники когнитивно-бихевиоральной терапии, арт-терапии, гештальт-терапии и ряд других психотерапевтических техник.

\section{Результаты и обсуждение:}

Известно, что в современной психотерапии в настоящее время весьма популярны идеи интеграции различных техник, которые неплохо взаимодополняют друг друга и усиливают эффект терапии [18].

Практические наблюдения показывают, что разнообразие применяемых терапевтических приемов и техник позволяет гибко реагировать на нестабильное психическое состояние пациентов с различными невротическими, аффективными и психосоматическими расстройствами.

Вследствие этого для проведения исследования был выбран интегративный (мультимодальный) психотерапевтический подход, включающий применение большого разнообразия техник и упражнений, позволяющих эффективно подействовать на разные составляющие тревожно-фобической симптоматики при СРК.

В качестве основных мишеней терапии у пациентов с тревожно-фобическими расстройствами при СРК были выбраны выявленные и свойственные для лиц из обследованной группы эмоциональноличностные особенности [10,12,14-17,19]:

$\square$ ипохондрическая фиксация на плохом самочувствии;

$\square$ разнообразные страхи (преимущественно по типу разной интенсивности канцерофобий); 


\begin{tabular}{|c|c|c|c|c|c|c|}
\hline Impact Factor: & $\begin{array}{l}\text { ISRA (India) } \\
\text { ISI (Dubai, UAE } \\
\text { GIF (Australia) } \\
\text { JIF }\end{array}$ & $\begin{array}{l}=1.344 \\
=0.829 \\
=0.564 \\
=1.500\end{array}$ & $\begin{array}{l}\text { SIS (USA) } \\
\text { PИНЦ (Russia) } \\
\text { ESJI (KZ) } \\
\text { SJIF (Morocco) }\end{array}$ & $\begin{array}{l}=0.912 \\
=0.234 \\
=1.042 \\
=2.031\end{array}$ & $\begin{array}{l}\text { ICV (Poland) } \\
\text { PIF (India) } \\
\text { IBI (India) }\end{array}$ & $\begin{array}{l}=6.630 \\
=1.940 \\
=4.260\end{array}$ \\
\hline
\end{tabular}

$\square$ мнительность, тревожные ожидания и опасения добавления к уже имеющимся проблемам еще каких-то дополнительных неприятностей и проблем извне;

$\square$ нестабильное аффективное состояние;

$\square$ нередко имеющаяся негативная оценка перспектив лечения;

$\square$ проблемная коммуникативная сфера, включающая обидчивость, следование нормативным социальным установкам долженствования, контроль над своим поведением в социуме;

$\square$ негибкость, ригидность жизненных установок, наполненных зачастую жесткими ограничивающими родительскими интроектами;

$\square$ проблемная самооценка с принижением себя;

$\square$ ощущение собственной беспомощности, ранимости, пребывание в бессильной, неспособной что-то поменять "жертвенной" роли;

$\square$ склонность к повышенной истощаемости таких пациентов.

Вследствие выявленных особенностей, послужившим основанием для определения целей терапии, были подобраны техники, помогающие как ресурсировать пациентов за достаточно непродолжительный срок времени (арт-терапия, трансовые ресурсные гипнотические техники), так и переключать с неприятных и дискомфортных ощущений, усиливающих тревогу, на более комфортное состояние (релаксационные техники, НЛП, позитивные визуализации), регулировать интенсивность своих тревог и страхов (аутотренинг, релаксация, приемы когнитивнобихевиоральной терапии), проработать жесткие и ригидные установки, (элементы когнитивнобихевиоральной терапии), наладить лучшую коммуникацию (некоторые техники трансактного анализа) или улучшить внутрисемейные отношения (элементы семейной терапия), повысить самооценку, уходя от позиции жертвы (транзактный анализ, гештальт-терапия, поведенческая терапия) с тренировкой менее "зажатого" и более раскованного поведения [10,12,14-17,19-22].

Естественно, значительный акцент в психотерапии был уделен основным для этой формы течения СРК психоэмоциональным проявлениям - тревогам и страхам.

В результате применения комбинированной терапии, включающей разнообразные психотерапевтические техники, ориентированные на купирование различных проявлений тревожно-фобических расстройств, а также прицельное воздействие на выбранные "мишени", можно было отметить практически во всей группе пациентов с СРК уже в первый месяц наблюдения и лечения существенное снижение тревожности, общее улучшение самочувствия, уменьшение/или исчезновение абдоминальных жалоб. Помимо субъективного, описываемого самими пациентами благополучия в дневниках самочувствия, такая же позитивная динамика наблюдалась и в ходе динамического врачебного осмотра. К концу 4-6 недели основного этапа терапии у пациентов наблюдались нормализация стула, стихали болевые проявления, уходили диспепсические явления и иные проявления со стороны ЖКТ, параллельно сопровождающие позитивные изменения в психоэмоциональной cфepe.

На этапе поддерживающей терапии можно было отметить продолжающееся улучшение и стабилизацию психоэмоционального состояния у всех пациентов отобранной группы с СРК с тревожно-фобической формой течения. Начиная с 8-10 недели терапии (совпавшей у большинства пациентов с концом второго месяца от начала терапии) при продолжении лечения было отмечено дальнейшее улучшение и стабилизация состояния как в психоэмоциональной, так и соматической сферах.

Заключение: Психотерапевтические занятия, основанные на использовании интегративного (мультимодального) подхода, в сочетании со стандартными гастроэнтерологическими методами терапии, проводимые в два этапа (1,5-месячное базовое лечение и 1,5-месячное поддерживающее лечение) показали достаточную терапевтическую эффективность в группе пациентов из 58 пациентов с СРК, имеющих тревожнофобическую симптоматику.

Комплексное использование психотерапевтических техник $\mathrm{c}$ включением суггестивных техник, элементов аутотренинга и приемов релаксации, нейро-линвистического программирования, и ряда других психотерапевтических приемов и техник, направляемых на выбранные терапевтические мишени при тревожно-фобическом варианте СРК, позволяет снизить проявления тревожных расстройств и существенно улучшить самочувствие пациентов (включая уменьшение абдоминальных жалоб) уже в первый месяц терапии. Поддерживающая терапия позволяет к концу второго месяца лечения стабилизировать и сделать устойчивым и благополучным как психоэмоциональное, так и соматическое состояние выбранной группы пациентов с СРК.

\section{References:}

ISPC Generalization of scientific results, 


\begin{tabular}{|c|c|c|c|c|c|c|}
\hline Impact Factor: & $\begin{array}{l}\text { ISRA (India) } \\
\text { ISI (Dubai, UAE } \\
\text { GIF (Australia) } \\
\text { JIF }\end{array}$ & $\begin{array}{l}=1.344 \\
=0.829 \\
=0.564 \\
=1.500\end{array}$ & $\begin{array}{l}\text { SIS (USA) } \\
\text { PИНЦ (Russia) } \\
\text { ESJI (KZ) } \\
\text { SJIF (Morocco) }\end{array}$ & $\begin{array}{l}=0.912 \\
=0.234 \\
=1.042 \\
=2.031\end{array}$ & $\begin{array}{l}\text { ICV (Poland) } \\
\text { PIF (India) } \\
\text { IBI (India) }\end{array}$ & $\begin{array}{l}=6.630 \\
=1.940 \\
=4.260\end{array}$ \\
\hline
\end{tabular}

1. Drossman DA (2006) The Functional Gastrointestinal Disorders and the Rome III Process//Gastroenterology. -2006. -130 (5). P.1377-1390.

2. Maev IV, Cheremushkin SV (2007) Sindrom razdrazhennogo kishechnika. Rimskie kriterii III // Gastroehnterologiya. (prilozhenie k zhurnalu Consilium Medicum).- 2007.- №1.- p. 29-33.

3. Ivashkin VT, Shelygin YA, Baranskaya EK, et al. (2014) Klinicheskie rekomendacii Rossijskoj gastroehnterologicheskoj associacii, Associacii koloproktologov Rossii po diagnostike i lecheniyu bol'nyh s sindromom razdrazhennogo kishechnika// Ross. zhurn. gastroehnterologii, gepatologii, koloproktologii. - 2014 № 2. - p. 92-101.

4. Goldberg J, Davidson P (1997) A biopsychosocial understanding of the irritable bowel syndrome: a review.// Can J Psychiatry. Oct 1997; Vol 42. -p.835-840.

5. Ivashkin VT, Poluehktova EA, Belkhusht S (2003) Sindrom razdrazhennogo kishechnika kak biopsikhosotsial'noe zabolevanie // Klinicheskie perspektivy $\mathrm{v}$ gastroehnterologii, gepatologii. - 2003. - N 6 . - p. 2-10

6. Makhov VM, Romasenko LV, Turko TV, et al. (2013) Mezhdistsiplinarnyj podkhod k sindromu razdrazhennogo kishechnika // Russkij meditsinskij zhurnal 2013. T.21, №13. p.702-704

7. Elfimova EV, Elfimov MA, Berezkin AS (2015) Psikhosomaticheskie vzaimootnosheniya pri zabolevaniyakh zheludochno-kishechnogo trakta na modeli sindroma razdrazhennogo kishechnika // Ehksperimental'naya i klin. gastroehnterologiya. 2015. №4 (116). - p. 8388.

8. Ford AC, Talley NJ, Schoenfeld PS, et al. (2009) Efficacy of antidepressants and psychological therapies in irritable bowel syndrome: systematic review and metaanalysis // Gut. - 2009. Mar. 58(3). - p.367-378.

9. Walker EA, Katon WJ, Jemelka RP, et al. (1992) Comorbidity of gastrointestinal complaints, depression, and anxiety in the Epidemiologic Catchment Area (ECA) Study // Am-JMed., 1992, Jan 24; 92(1A) - p.26-30.

10. Marilov VV, Korkina MV, Esaulov VI (2000) Lichnostnye osobennosti i kharakter psikhicheskikh narushenij pri sindrome razdrazheniya tolstoj kishki // Sotsial'naya i klinicheskaya psikhiatriya. - 2000. - T. 10, № 4. - p. 21-27.

11. Roganov DA (2005) Affektivnye rasstrojstva pri sindrome razdrazhennogo kishechnika (klinicheskie osobennosti, diagnostika, lechenie) : avtoref. dis ... kand. med. nauk. M., 2005. $-25 \mathrm{p}$.
12. Marilov VV, Esaulov VI (2000) Psihicheskie narusheniya pri sindrome vozbudimoj tolstoj kishki//Vestnik RUDN. Seriya: Medicina. 2000. -№ 3. -p. 98-101.

13. Kozlova IV, Myalina YN, Lekareva LI, et al. (2014) Psihologicheskie osobennosti pacientov s funkcional'nymi i vospalitel'nymi zabolevaniyami kishechnika // Saratovskij nauchno-medicinskij zhurnal. - 2014. - T. 10, № 1. - p.80-85.

14. Esaulov VI (2015) O nekotoryh ehmocional'nolichnostnyh harakteristikah pacientov $\mathrm{s}$ sindromom razdrazhennogo kishechnika. European research. -2015. -№ 5 (6). -p.76-78.

15. Esaulov VI (2016) The experience of тне application of psychotherapeutic techniques in the treatment of borderline mental disorders in patients with irritable bowel syndrome. ISJ Theoretical \& Applied Science, 10 (42): 77-86. Soi: http://s-o-i.org/1.1/TAS-10-42-17 Doi: http://dx.doi.org/10.15863/TAS.2016.10.42.17

16. Esaulov VI, Marilov VV (2001) Kompleksnaya psihoterapiya sindroma razdrazhennogo kishechnika//Vestnik RUDN. Seriya: Medicina. - 2001. -№ 2. -p.54-57.

17. Esaulov VI (2016) Opyt primeneniya razlichnyh psihoterapevticheskih tekhnik $\mathrm{v}$ lechenii pacientov $\mathrm{s}$ sindromom razdrazhennogo kishechnika // Sovremennye problemy nauki i obrazovaniya. -2016. -№ 6.; Available: https://scienceeducation.ru/ru/article/view? $\mathrm{id}=25468$ (Accessed: 30.12.2016).

18. Makarov VV (2001) Psihoterapiya novogo veka. M., Akademicheskij proekt .-2001.-496 p.

19. Esaulov VI (2016) Ob opyte primeneniya u bol'nyh $\mathrm{s}$ sindromom razdrazhennogo kishechnika metodov kompleksnoj psihodiagnostiki //Sovremennye problemy nauki i obrazovaniya. - 2016. - № 4. DOI:10.17513/spno.24911

20. Pahomova IV, Ajvazyan TA, Zajcev VP, et al. (2008) Autogennaya trenirovka v vosstanovitel'nom lechenii bol'nyh s sindromom razdrazhennogo kishechnika s preobladaniem zaporov // Voprosy kurortologii, fizioterapii i lechebnoj fizicheskoj kul'tury. - 2008. - №1. p.24-27.

21. Esaulov VI (2015) Ob opyte primeneniya relaksacionnyh metodov $\mathrm{v}$ lechenii pacientov s sindromom razdrazhennogo kishechnika // Vestnik nevrologii, psihiatrii, nejrohirurgii 2015. № 10. -p. 60-64

22. Esaulov VI (2016) Primenenie abdominal'nogo varianta gipnoza $\mathrm{v}$ kompleksnom lechenii sindroma razdrazhennogo kishechnika // Vestn. Novg. gos. un-ta. Ser.: Medicinskie nauki. 2016. № 1(92). -p.90-94. 